Gefässchirurgie 2016 $\cdot 21: 538-539$

DOI 10.1007/s00772-016-0207-x

(c) Springer-Verlag Berlin Heidelberg 2016

CrossMark

\author{
I. Flessenkämper' $\cdot$ A. Mumme ${ }^{2}$ \\ 'Klinikum rechts der Isar der Technischen Universität München, München, Deutschland \\ ${ }^{2}$ St. Josef-Hospital im Katholischen Klinikum Bochum, Bochum, Deutschland
}

\title{
Seltene Erkrankungen der Venen
}

Venöse Erkrankungen sind an sich wesentlich häufiger als arterielle Erkrankungen. In deutschen Krankenhäusern werden etwa dreimal mehr Venen als Arterien versorgt. Die Hauptbereiche betreffen Varizen, Thrombophlebitiden und tiefe Venenthrombosen. Diese Tatsache soll aber nicht den Blick darauf verstellen, dass es in diesem Bereich auch Pathologien und Themen gibt, die zwar weniger häufig sind, aber nichtsdestotrotz von immanenter Bedeutung für die Betroffenen sein können. Der gefäßmedizinische Diagnostiker und Therapeut muss das Wissen um diese Erkrankungen und den Umgang mit ihnen parat haben. Er muss sie zumindest erkennen und managen können.

Einige ausgesuchte Kapitel möchten wir Ihnen als Schwerpunkt in diesem Heft präsentieren. Hierbei werden wie im ersten Beitrag von Jörg Tessarek über Interventionen bei Tumorkompression der großen Venen/suprakardialen Venen auch Nachbardisziplinen - wie etwa die Onkologie - berührt. Jeder, der mit der Problematik der oberen Einflussstauung befasst ist, weiß, wie elementar das Leiden für die hiervon Betroffenen ist und wie hilfreich auch eine palliative Erleichterung für diese Menschen sein kann, selbst wenn es oft nur für eine kurze Zeitspanne der Gesamterkrankung sein kann.

Diagnostik und Therapie sind das eine. Die langfristige Sekundärbehandlung ist ein weiteres Element, von dem der Erfolg medizinischer Handlungen im venösen Bereich abhängt. Die Antikoagulation und Hämostaseologie hat hier - wie in der gesamten Gefäßmedizin - eine herausragende Bedeutung, vor allem wenn die Umstände des Patienten so gar nicht zu den Leitlinien passen wollen. Was ist, wenn auf eine Secondline-Therapie zurückgegriffen werden muss? Fast täglich stellen sich jedem von uns hierzu Fragen, die nicht immer leicht zu beantworten sind. Hiermit beschäftigt sich Hinrich Böhner in seinem Beitrag über ASS und Venen. Wie so oft bei seltenen Erkrankungen und Konstellationen, stellt die viel gerühmte Evidenz hier ein eigenes Problem dar, da sie in gewünschter Klarheit nicht existiert. Im pharmakologischen Bereich gibt es aber Ansätze, allmählich zu verlässlichen Daten zu kommen.

In dem Artikel über venöse Aneurysmen von Ingo Flessenkämper wird diese teils wenig bekannte Morphologie vorgestellt und in ihrer Wertigkeit begutachtet. Hier geht es um ein Krankheitsbild, das zwar recht selten ist, aber in seiner Gefährlichkeit oft unterschätzt wird. Ein Krankheitsbild, das ehemals fast ausschließlich über den Umweg einer Lungenarterienembolie diagnostiziert wurde.

In dem Beitrag von Thomas Bürger zur V.-subclavia-Thrombose bei ,thoracic inlet syndrome" kommen die breiten Erfahrungen des Autors im Umgang mit dieser Kombination mehrerer miteinander zusammenhängender Faktoren und Symptome zum Tragen. Da die Zeit hier oftmals drängt, steht es auch jedem Gefäßmediziner, der nicht täglich mit diesem Krankheitsbild konfrontiert wird, gut zu Gesicht, sein Wissen zu vertiefen und im Ernstfall parat zu haben.

Last, but not least trägt Achim Mumme mit der Darstellung seiner großen Erfahrung im Management der Phlegmasia coerulea dolens zum Kanon der seltenen Erkrankungen in der Phlebologie bei. Auch hier handelt es sich um ein Krankheitsbild, dessen sofortige Therapie obligat ist, will man nicht das Leben der Patienten oder eventuell den Verlust einer Extremität in Kauf nehmen. 
Wir stellen Ihnen Aspekte der Phlebologie vor, für die es keine Evidenz gibt, ja nicht geben kann, da quantitativ keine wirklich belastbare Statistik erreichbar ist. Von prospektiv randomisierten Studien zur Evaluierung verschiedener Therapieansätze kann man hier nicht einmal träumen. Deshalb muss zur bestmöglichen Betreuung und Beratung der Patienten Wissen zusammengetragen werden, damit es von den dann behandelnden Ärzten in ein Verhältnis zu den individuellen Umständen des Patienten gesetzt, abgewogen und genauso wie geschildert oder in Variationen angewandt werden kann. Hierzu wollen wir für die seltenen venösen Erkrankungen und Konstellationen mit diesem Heft beitragen.

Vielleicht bringt Ihnen die Beschäftigung mit den in diesem Heft dargestellten venösen Erkrankungen nicht nur neue Erkenntnisse, sondern vermag auch den Blick auf die Phlebologie als einem spannenden Kapitel der Gefäßmedizin schärfen, das weit über die lohnende Versorgung von Varizen hinausgeht.

Wir wünschen Ihnen eine spannende Lektüre.

Ihre

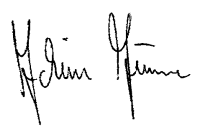

Prof. Achim Mumme

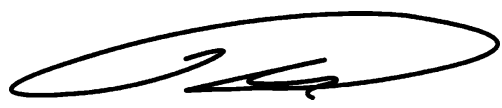

Dr. Ingo Flessenkämper

\section{Korrespondenzadresse}

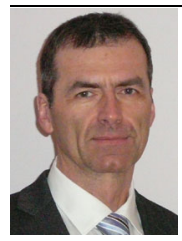

Dr. I. Flessenkämper

Klinikum rechts der Isar der Technischen Universität München

Ismaninger Str. 22,

81675 München, Deutschland drifless@t-online.de

Interessenkonflikt. I. Flessenkämper und A. Mumme geben an, dass kein Interessenkonflikt besteht.

\section{Wissenschaftsrat will Universitätsmedizin zukunftsfähig machen}

Auf die besondere Bedeutung der Universitätsmedizin als „Fundament des Gesundheitssystems" hat der Wissenschaftsrat (WR) hingewiesen. In einer neuen Expertise gibt er Empfehlungen zur Stärkung der universitären Medizin.

Mit den „Perspektiven der Universitätsmedizin" legt der Wissenschaftsrat ein umfassendes Programm zur Weiterentwicklung der universitären Medizin in den nächsten zehn bis fünfzehn Jahren vor. Seine Empfehlungen richten sich auf die Rahmenbedingungen und Ressourcen, die die Universitätsmedizin benötigt, um ihren Aufgaben in Forschung, Lehre und Krankenversorgung auch künftig gerecht werden zu können. Der Wissenschaftsrat empfiehlt zwei Strategien, die parallel umgesetzt werden sollten: den Ausbau bestehender Strukturen an allen universitätsmedizinischen Standorten und den Aufbau vollständig neuer Bereiche, die jungen Wissenschaftlern und Ärzten besondere Perspektiven bieten, so genannte "Profilbereiche". Dazu gehörten verbesserte Rahmenbedingungen für den wissenschaftlichen Nachwuchs, insbesondere in der klinischen Forschung. Unterschiedliche Gruppen von Wissenschaftlern benötigten strukturierte Karrierewege und attraktive berufliche Ziele auch neben der Professur. Handlungsbedarf sieht der Wissenschaftsrat außerdem bei der Qualitätssicherung von Forschung und Lehre und bei der Weiterentwicklung von forschungs- und informationstechnologischen Infrastrukturen. Auch sollte das innovative Potenzial der Universitätsmedizin in größerem Umfang als bisher auf allen Ebenen der Krankenversorgung nutzbar gemacht werden. Diese Maßnahmen werden aber nicht ausreichen, um die Zukunftsfähigkeit der deutschen Universitätsmedizin im internationalen Wettbewerb sicherzustellen, warnt der Rat.

\section{Doppelgleisiger Ausbau}

Er empfiehlt daher als neuen Ansatz den Aufbau von Profilbereichen in der Universitätsmedizin. Diese Organisationsstrukturen sollen die klassische Fächeraufteilung in der Medizin überwinden und eine Spezialisierung in Forschung und Krankenversorgung auf internationalem Spitzenniveau ermöglichen. Die neuen Profilbereiche sollen spezifischen Anforderungen unter anderem an Forschungs- und Translationsleistun- gen, Weiterbildungsstrukturen, Karrierewege und -ziele für den wissenschaftlichen Nachwuchs sowie an die Qualitätssicherung von Forschung und Versorgung genügen. Regelmäßige Evaluationen mit Konsequenzen für die Ausstattung sind unabdingbar, so die Vorstellung des Wissenschaftsrats. Er fordert dafür kreative Konzepte für innovative Organisationsstrukturen, die sich im Wettbewerb als zukunftsweisend qualifizieren. „Erfolgreiche Profilbereiche können und sollen als Nukleus für weitergehende Reformprozesse in der Universitätsmedizin wirksam werden." Angesichts der Systemrelevanz der universitären Medizin für das deutsche Gesundheitssystem betrachtet der Wissenschaftsrat die Förderung von Profilbereichen als eine gemeinsame Aufgabe von Bund und Ländern.

Quelle: Wissenschaftsrat, www.wissenschaftsrat.de 